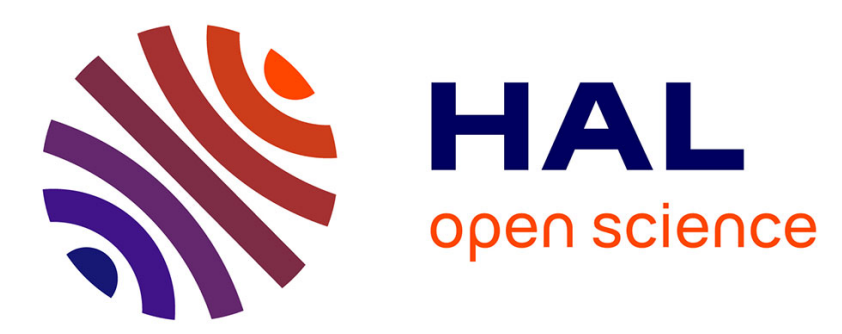

\title{
A nonlinear weakly dispersive method for recovering the elevation of irrotational surface waves from pressure measurements
}

\author{
Philippe Bonneton, David Lannes, Kévin Martins, Hervé Michallet
}

\section{- To cite this version:}

Philippe Bonneton, David Lannes, Kévin Martins, Hervé Michallet. A nonlinear weakly dispersive method for recovering the elevation of irrotational surface waves from pressure measurements. Coastal Engineering, 2018, 138, pp.1 - 8. 10.1016/j.coastaleng.2018.04.005 . hal-01778147

\section{HAL Id: hal-01778147 \\ https://hal.science/hal-01778147}

Submitted on 25 Apr 2018

HAL is a multi-disciplinary open access archive for the deposit and dissemination of scientific research documents, whether they are published or not. The documents may come from teaching and research institutions in France or abroad, or from public or private research centers.
L'archive ouverte pluridisciplinaire HAL, est destinée au dépôt et à la diffusion de documents scientifiques de niveau recherche, publiés ou non, émanant des établissements d'enseignement et de recherche français ou étrangers, des laboratoires publics ou privés. 


\title{
A nonlinear weakly dispersive method for recovering the elevation of irrotational surface waves from pressure measurements
}

\author{
Bonneton P. \\ University of Bordeaux ; CNRS ; UMR 5805 EPOC, Allée Geoffroy Saint-Hilaire, F-33615 \\ Pessac, France \\ Lannes D. \\ University of Bordeaux; IMB and CNRS UMR 5251, Talence, F-33405 France \\ Martins K. \\ UMR 7266 LIENSs, CNRS-Université de La Rochelle, 2 rue Olympe de Gouges, La \\ Rochelle, 17000, France \\ Michallet $\mathrm{H}$. \\ University Grenoble Alpes, CNRS, LEGI, CS40700, 38058 Grenoble, France
}

\begin{abstract}
We present the derivation of a nonlinear weakly dispersive formula to reconstruct, from pressure measurements, the surface elevation of nonlinear waves propagating in shallow water. The formula is simple and easy to use as it is local in time and only involves first and second order time derivatives of the measured pressure. This novel approach is evaluated on laboratory and field data of shoaling waves near the breaking point. Unlike linear methods, the nonlinear formula is able to reproduce at the individual wave scale the peaked and skewed shape of nonlinear waves close to the breaking point. Improvements in the frequency domain are also observed as the new method is able to accurately predict surface wave elevation spectra over four harmonics. The nonlinear weakly dispersive formula derived in this paper represents an economic and easy
\end{abstract}

\footnotetext{
${ }^{*}$ Corresponding author

Email address: philippe.bonneton@u-bordeaux.fr (Bonneton P.)
} 
to use alternative to direct wave elevation measurement methods (e.g. acoustic surface tracking and LiDAR scanning)..

Keywords: surface wave, measurements, nonlinear, weakly dispersive, nonhydrostatic, shallow water, LiDAR, Acoustic Surface Tracking

\section{Introduction}

Near-bottom-mounted pressure sensors have long been used for measuring surface wave in the nearshore. However, the relationship between bottom pressure and sea surface elevation is not straightforward. This relationship is com5 monly assumed to be given by linear wave theory, the so-called transfer function method (e.g. Bishop and Donelan [4] and Tsai et al. [28]). The validity of this linear reconstruction has been extensively studied in field conditions for waves propagating in relatively shallow water [14, 11, 7, 12]. Although discrepancies were greater close to the break point, Guza and Thornton [12] found a good agreement in and outside the surf zone between sea surface elevation spectra derived from pressure data and from direct elevation measurements. Errors in both total variance and energy density in a particular frequency band were less than 20\%. In a more controlled environment, Bishop and Donelan [4] estimated that using linear wave theory was leading to error of about $5 \%$ of the wave height; uncertainty in the deployment of in situ instruments and the data itself was thought to be responsible for the varying error estimates found in the literature. Following these seminal studies, the linear reconstruction method has become the main approach for characterizing shallow-water surface-wave elevation in field conditions.

This approach is commonly used for determining bulk wave parameters such as the significant wave height $H_{s}$, but it has also served as a basis for studying nonlinear wave interactions in the field (e.g. Elgar and Guza [9], Elgar et al. [10], Senechal et al. [26], Henderson et al. [13]). However, we know that wave nonlinearities can be strong in the shoaling zone, especially in the region close 25 to the onset of breaking, and thus the use of a linear theory to reconstruct 
wave elevation can be questioned. For instance, Bonneton and Lannes [6] and Martins et al. [19] have shown that the linear reconstruction fails to describe the peaky and skewed shape of nonlinear waves, and lead to an underestimation of the individual wave height by up to $30 \%$ just prior the breaking point [19].

30 Such measurement errors are problematic for many coastal applications, such as studies on wave overtopping and submersion which require accurate measurements of the highest wave crests. Furthermore, a correct description of wave asymmetry and skewness is of paramount importance for understanding sediment dynamics (e.g. Dubarbier et al. [8]). Finally, an accurate description of the wave elevation field is also crucial for the validation of the new generation of fully-nonlinear phase-resolving wave models (e.g. Zijlema et al. [30], Bonneton et al. [5] or Shi et al. [27]).

Even if some methods are now available for a direct measurement of the surface elevation, such as acoustic surface tracking [3] or LiDAR scanning [18],

40 pressures sensors remain a very useful tool for coastal wave applications. Indeed, they are cheap, robust, not sensitive to air bubbles or turbidity, and easy to deploy since they do not require the presence of nearshore infrastructure, as it can be the case for LiDAR technology [e.g., see 21]. Bonneton and Lannes [6] recently derived a method which allows a fully dispersive nonlinear recon45 struction of the surface elevation from pressure measurements. Comparisons with numerical Euler solutions and laboratory data showed that this nonlinear method provides much better results than the classical linear approach. It gives an accurate prediction of the maximum elevation and, contrary to the nonlinear heuristic method proposed by Oliveras et al. [24], it accurately reproduces the skewed shape of nonlinear dispersive wave fields. However, this method requires, like the classical linear transfer approach [4, 28] and the heuristic method [24, 29], the use of a frequency cutoff which becomes a limiting factor for the reconstruction of strongly nonlinear waves. In the present paper we derive a nonlinear weakly-dispersive method which allows an accurate reconstruction of nonlinear waves in shallow water, especially just prior to breaking. 


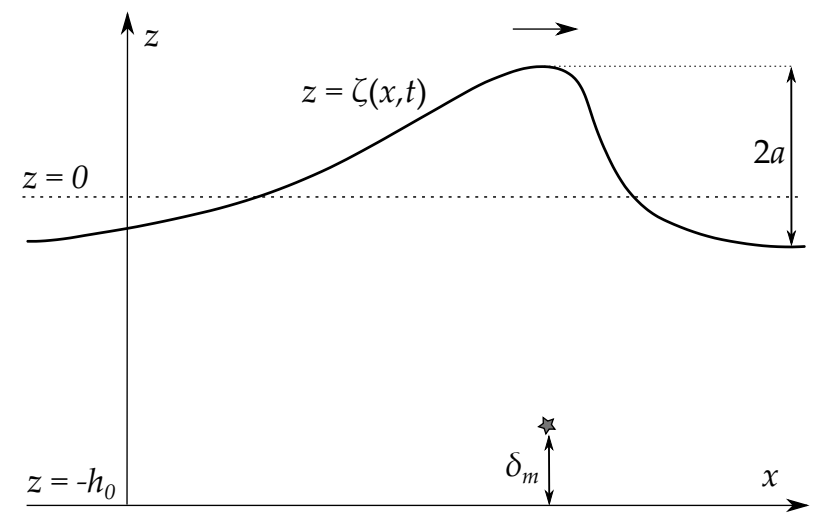

Figure 1: Sketch of the Cartesian coordinate system: $x$ is the horizontal axis along which waves propagate and $z$ points vertically upwards, with $z=0$ being the mean water level and $z=-h_{0}$ the distance to the bottom. The wave amplitude is noted $a$ and $\delta_{m}$ represents the distance to the bottom at which the pressure is measured.

\section{Nonlinear weakly-dispersive reconstruction formula}

In this section we derive a formula working in the time domain, which allows the elevation reconstruction of nonlinear shallow water waves from pressure measurements. This formula is an approximate expression, in the shallow water regime, of the fully dispersive formula derived in [6]. The derivation presented in this section is much simpler and straightforward compared to the general fully dispersive derivation.

We consider that the wave field is locally close to a two-dimensional wave field. We choose Cartesian coordinates $(x, z)$, where $x$ is the horizontal axis along which waves propagate and $z$ the upward vertical coordinate. We denote $z=\zeta(x, t)$ the elevation of the free surface above the still water level $z=0$, and by $z=-h_{0}$ the constant bottom elevation (see Figure 1). The water depth $h$ can be expressed as $h(x, t)=h_{0}+\zeta(x, t)$. The pressure $P_{\mathrm{m}}$ is measured at a distance $\delta_{m}$ above the bottom, $P_{\mathrm{m}}=P_{\left.\right|_{z=-h_{0}+\delta_{m}}}$, where $P(x, z, t)$ is the 70 pressure field.

The fluid motion is governed by the free-surface incompressible irrotational Euler equations; if the flow is irrotational, as it is assumed here, it is conve- 
nient to work with a velocity potential instead of the velocity field, and with Bernoulli's equation instead of Euler equations. If $\phi$ denotes the velocity potential, these equations can be recast in the form:

$$
\begin{aligned}
\partial_{x}^{2} \phi+\partial_{z}^{2} \phi & =0 \\
\partial_{t} \phi+g z+\frac{1}{2}\left|\partial_{x} \phi\right|^{2}+\frac{1}{2}\left|\partial_{z} \phi\right|^{2} & =-\frac{1}{\rho}\left(P-P_{a t m}\right),
\end{aligned}
$$

where $\rho$ is the water density, $g$ the gravity and $P_{a t m}$ the (constant) atmospheric pressure. These equations are complemented by boundary conditions. At the bottom we have

$$
\partial_{z} \phi=0 \quad \text { on } z=-h_{0} ;
$$

at the surface, we have the classical kinematic equation on $\zeta$,

$$
\partial_{t} \zeta=\partial_{z} \phi-\partial_{x} \zeta \cdot \partial_{x} \phi \quad \text { on } \quad z=\zeta
$$

and the pressure continuity,

$$
P=P_{\mathrm{atm}} \quad \text { on } \quad z=\zeta .
$$

Three main length scales are involved in this problem: the characteristic horizontal length $L$ ( $L=1 / k$, where $k$ is the typical wave number), the amplitude $a$ of the wave, and the depth at rest $h_{0}$. The problem is then controlled by two dimensionless parameters:

$$
\varepsilon=\frac{a}{h_{0}}, \quad \mu=\frac{h_{0}^{2}}{L^{2}}=\left(h_{0} k\right)^{2}
$$

where $\varepsilon$ is a nonlinearity parameter while $\mu$ is the shallowness parameter. The different variables and functions involved in this problem can be put in dimensionless form using the relations

$$
\begin{aligned}
x^{\prime} & =\frac{x}{L}, \quad z^{\prime}=\frac{z}{h_{0}}, \quad \delta_{m}^{\prime}=\frac{\delta_{m}}{h_{0}}, \quad t^{\prime}=\frac{\sqrt{g h_{0}}}{L} t, \\
\zeta^{\prime} & =\frac{\zeta}{a}, \quad h^{\prime}=\frac{h}{h_{0}}=1+\epsilon \zeta^{\prime}, \quad \phi^{\prime}=\frac{h_{0}}{a L \sqrt{g h_{0}}} \phi, \quad P^{\prime}=\frac{P}{\rho g h_{0}},
\end{aligned}
$$

where the primes are used to denote dimensionless quantities.

Omitting the primes for the sake of clarity, the vertical momentum equation, in 
dimensionless form, writes

$$
\varepsilon \Gamma=-1-\partial_{z} P,
$$

where $\Gamma=\partial_{t} w+\varepsilon u \partial_{x} w+\frac{\varepsilon}{\mu} w \partial_{z} w$ is the vertical acceleration, $u=\partial_{x} \phi$ the horizontal velocity and $w=\partial_{z} \phi$ the vertical velocity. Integrating this equation over $z$ we get

$$
\zeta=\zeta_{\mathrm{H}}-\int_{-1+\delta_{m}}^{\varepsilon \zeta} \Gamma d z
$$

where $\zeta_{\mathrm{H}}$ is the dimensionless hydrostatic reconstruction

$$
\zeta_{\mathrm{H}}=\frac{1}{\varepsilon}\left(P_{\mathrm{m}}-P_{\mathrm{atm}}-1+\delta_{m}\right) .
$$

The formula (4) is exact but involves quantities that cannot be expressed in terms of the measured pressure $P_{\mathrm{m}}$. Our goal is to derive approximate formulas that can be expressed as a function of $P_{\mathrm{m}}$, or equivalently $\zeta_{\mathrm{H}}$. Following [16], we shall perform an asymptotic expansion of (4) in terms of the shallowness parameter $\mu$.

The velocity potential $\phi$ is given at second order by

$$
\phi=\psi-\frac{\mu}{2}\left((z+1)^{2}-h^{2}\right) \partial_{x}^{2} \psi+O\left(\mu^{2}\right),
$$

with $\psi=\phi_{\mid z=\varepsilon \zeta}$. From this equation we can deduce that

$$
\begin{aligned}
u & =U+O(\mu) \\
w & =-\mu(z+1) \partial_{x} U+O\left(\mu^{2}\right),
\end{aligned}
$$

where $U$ is the depth-averaged horizontal velocity. Using these relations Eq. (4) becomes

$$
\zeta=\zeta_{H}-\frac{\mu}{2}\left(h^{2}-\delta_{m}^{2}\right)\left(\partial_{x}^{2} \zeta+2 \epsilon\left(\partial_{x} U\right)^{2}\right)+O\left(\mu^{2}\right) .
$$

From the linearized water waves equations in shallow water, we know that $\partial_{t}^{2} \zeta-$ $\partial_{x}^{2} \zeta=O(\varepsilon, \mu)$, and the above formula therefore yields

$$
\partial_{x}^{2} \zeta_{H}=\partial_{t}^{2} \zeta_{H}+O(\varepsilon, \mu)
$$

For weakly nonlinear waves $(\varepsilon=O(\mu))$ we deduce a linear shallow water reconstruction formula from (77) and (8),

$$
\zeta_{\mathrm{SL}}=\zeta_{\mathrm{H}}-\frac{\mu}{2}\left(1-\delta_{m}^{2}\right) \partial_{t}^{2} \zeta_{\mathrm{H}}
$$


which is valid up to terms of order $O\left(\varepsilon \mu, \mu^{2}\right)$.

For moderately nonlinear waves $\left(\varepsilon=O(\mu)^{1 / 2}\right)$ the $O(\varepsilon \mu)$ terms cannot be neglected, and we therefore seek a higher order correction of (9) under the form

$$
\zeta_{\mathrm{SNL}}=\zeta_{\mathrm{SL}}+\varepsilon \mu C
$$

where the corrector term $C$ is chosen in such a way that Eq. (7) is satisfied up to $O\left(\mu^{2}\right)$ terms if $\zeta$ is replaced by $\zeta_{\mathrm{SNL}}$. This yields the condition

$$
-\frac{\mu}{2}\left(1-\delta_{m}^{2}\right)\left(\partial_{t}^{2} \zeta_{\mathrm{H}}-\partial_{x}^{2} \zeta_{\mathrm{H}}\right)+\varepsilon \mu C=-\varepsilon \mu\left(\zeta \partial_{x}^{2} \zeta+\left(1-\delta_{m}^{2}\right)\left(\partial_{x} U\right)^{2}\right)+O\left(\mu^{2}\right)
$$

From the mass conservation equation $\partial_{t} \zeta+\partial_{x}(h U)=0$, we deduce that $\partial_{x} U=$ $-\partial_{t} \zeta+O(\varepsilon)$ which, together with (8) yields

$$
\partial_{t}^{2} \zeta_{\mathrm{H}}-\partial_{x}^{2} \zeta_{\mathrm{H}}=\frac{2 \varepsilon}{1-\delta_{m}^{2}}\left(C+\partial_{t}\left(\zeta \partial_{t} \zeta\right)-\delta_{m}^{2}\left(\partial_{t} \zeta\right)^{2}\right)+O(\mu) .
$$

The $O(\varepsilon)$ terms gathers the quadratic interactions of the wave field. Since at order $O(\varepsilon, \mu)$, the waves are governed by a linear wave equation with speed 1 , these quadratic terms are either the product of co-propagating or counterpropagating waves and therefore of the form

$$
\partial_{t}^{2} \zeta_{\mathrm{H}}-\partial_{x}^{2} \zeta_{\mathrm{H}}=\varepsilon(F(x-t)+G(x+t)+\mathcal{F}(x-t) \mathcal{G}(x+t))+O(\mu),
$$

for some functions $F$ and $G$ that depend quadratically on $\zeta$ and some functions $\mathcal{F}$ and $\mathcal{G}$ that depend linearly on $\zeta$.

The only possibility that does not create secular growth for $\zeta_{\mathrm{H}}$ is if the righthand-side is equal to zero (see for instance [15] or Lemma 7.20 in 17]). This corresponds to

$$
C=-\partial_{t}\left(\zeta \partial_{t} \zeta\right)+\delta_{m}^{2}\left(\partial_{t} \zeta\right)^{2}
$$

Since $\zeta=\zeta_{\mathrm{SL}}+O(\mu)$, we therefore obtain the following nonlinear shallow water reconstruction formula

$$
\zeta_{\mathrm{SNL}}=\zeta_{\mathrm{SL}}-\varepsilon \mu\left(\partial_{t}\left(\zeta_{\mathrm{SL}} \partial_{t} \zeta_{\mathrm{SL}}\right)-\delta_{m}^{2}\left(\partial_{t} \zeta_{\mathrm{SL}}\right)^{2}\right)
$$

which is valid up to $O\left(\mu^{2}\right)$ terms for moderately nonlinear waves. A generalization of this formula in presence of a background current is given in 6 ]. 
The reconstruction formula (10) has been derived under the assumption of a locally flat bottom. We can easily extend this approach by taking into account a slowly varying bathymetry. The bottom is given by $z=-h_{0}+b(x)$, where $b$ is a slowly varying function of $x$. The nonlinear reconstruction becomes in dimensionless form

$$
\zeta=\zeta_{\mathrm{SNL}}+\sqrt{\mu} \sigma_{b} \partial_{x} b \partial_{x} \zeta+O\left(\mu^{2}, \mu \sigma_{b}\right)
$$

where $\sigma_{b}$ is the characteristic slope of the bathymetry at the measurement location. For unidirectional traveling waves the nonlinear reconstruction simplifies to the form which follows

$$
\zeta=\zeta_{\mathrm{SNL}}-\sqrt{\mu} \sigma_{b} \partial_{x} b \partial_{t} \zeta_{\mathrm{SNL}}+O\left(\mu^{2}, \mu \sigma_{b}\right) .
$$

The parameter $\sqrt{\mu} \sigma_{b}$ being very small for many coastal applications, as those discussed in section 3, we neglect throughout the paper the bottom contribution.

In variables with dimension the reconstruction formulas (5), (9) and (10) become

$$
\begin{aligned}
\zeta_{\mathrm{H}} & =\frac{P_{\mathrm{m}}-P_{\mathrm{atm}}}{\rho g}-h_{0}+\delta_{m} \\
\zeta_{\mathrm{SL}} & =\zeta_{\mathrm{H}}-\frac{h_{0}}{2 g}\left(1-\left(\delta_{m} / h_{0}\right)^{2}\right) \partial_{t}^{2} \zeta_{\mathrm{H}} \\
\zeta_{\mathrm{SNL}} & =\zeta_{\mathrm{SL}}-\frac{1}{g}\left(\partial_{t}\left(\zeta_{\mathrm{SL}} \partial_{t} \zeta_{\mathrm{SL}}\right)-\left(\delta_{m} / h_{0}\right)^{2}\left(\partial_{t} \zeta_{\mathrm{SL}}\right)^{2}\right) .
\end{aligned}
$$

Contrary to fully dispersive reconstructions (see 6] ) these formulas can be applied locally in time and do not necessarily require Fourier transforms (see Appendix A). The ability of these formulas to reconstruct shallow water waves was first assessed by [6] from comparison with solitary wave solutions computed from the Euler equations. In the next section the validation is extended to nonlinear shallow water waves propagating on gently sloping bottoms.

\section{Applications to laboratory and field data}

To assess the ability of the formulas derived in the preceding section we use laboratory and field data corresponding to weakly dispersive waves $(\mu \leq 0.28)$ 
propagating in the shoaling zone close to the breaking point. Such test cases, which are associated with strong nonlinearities (i.e. large $\epsilon$ ), represent highly flume, Vollenhove, The Netherlands. Martins et al. [19] used pressure and sur- 
face elevation data obtained from a LiDAR scanner from this test to validate the simulated wave transformation across the wave flume. Although the datasets are described in Martins et al. [19], some basic information are reminded here.

Second-order monochromatic Stokes waves $(T=12.1 \mathrm{~s}, H=0.68 \mathrm{~m})$ were generated in the experimental flume. The pressure was measured just before the break point, with a sampling frequency of $20 \mathrm{~Hz}$. The pressure sensor was situated at a distance $\delta_{m}=0.33 \mathrm{~m}$ above the bottom. For this wave test, the still water depth was $h_{0}=1.17 \mathrm{~m}$, the characteristic bottom slope was $\sigma_{b}=0.041$ and the values of the dimensionless parameters were $\mu=0.032$ and $\varepsilon=0.65$. It is worth noting that the pressure data was obtained close to the breaking point [19], where nonlinearities are the strongest and close to a crest antinode, due to the strong reflection experienced during the test.

The LiDAR scanner consisted of a LMS511 SiCK eye-safe $(\lambda=905 \mathrm{~nm})$ 2D scanner deployed $3.9 \mathrm{~m}$ above the still water level, a meter landward of the pressure transducer. Unlike the pressure transducer, the scanner directly detects the surface by capturing back the light beam scattered at the surface by the presence of ripples/roughness or air bubbles in case of breaking. The LiDAR data were collected at $37.5 \mathrm{~Hz}$, and only the data at the pressure transducer location is used. For this specific experiment the LiDAR measurement accuracy was about $0.02 \mathrm{~m}$. The reader is referred to Martins et al. [18, 19] for more details on the scanner data processing.

Figure 2 presents a comparison, over four wave periods, between reconstructed wave elevation and direct LiDAR measurements. We can see that in this shallow water regime the linear reconstruction (12) brings little improvement in comparison with the hydrostatic formula (11) and strongly underestimates the crest elevation. A wave by wave analysis shows that the root mean square (RMS) error for the wave height estimated from the linear reconstruction reaches $28 \%$ (see also [19]). By contrast, the nonlinear formula (13) gives much better results. In particular, we can see in Figure 3 that the peaked and skewed shape of the wave is much better described by the nonlinear formulation Eq. (13) than by the linear one Eq. (12). In order to quantify the wave asymmetry 


\begin{tabular}{|l|c|c|c|c|}
\hline & $\zeta_{\mathrm{H}}$, eq. (11) & $\zeta_{\mathrm{SL}}$, eq. (12) & $\zeta_{\mathrm{SNL}}$, eq. (13) & $\zeta_{\mathrm{LiDAR}}$ \\
\hline$S_{k}$ & 0.98 & 1.12 & 1.46 & 1.45 \\
\hline$S_{k}$ error & $32.2 \%$ & $22.5 \%$ & $0.7 \%$ & - \\
\hline
\end{tabular}

Table 1: Sea surface skewness. Comparison between reconstructed elevation and direct LiDAR elevation measurements.

with respect to the horizontal axis we have computed the skewness parameter:

$$
S_{k}=\frac{\left\langle(\zeta-\langle\zeta\rangle)^{3}\right\rangle}{\left\langle(\zeta-\langle\zeta\rangle)^{2}\right\rangle^{3 / 2}}
$$

where $\langle$.$\rangle is the time-averaging operator. Table 1$ shows that the linear reconstruction strongly underestimates, by $22.5 \%$, the wave skewness. By contrast the skewness is very well reproduced by the nonlinear reconstruction with an error, for this specific test case, smaller than $1 \%$. The nonlinear formula also improves the evaluation of the wave crest elevation (RMS error of 17\%), but less significantly compared with the skewness.

The skewness parameter is related to the velocity skewness at the bed (see e.g. Rocha et al. 25]) which, along with the wave asymmetry, can be used to compute the sediment transport rate (e.g. Abreu et al. [1]). The wave high order harmonics also contribute to the acceleration effects that can promote bed motion (e.g. Berni et al. [2]). This underlines the need to accurately characterize wave non-linearities for predicting sediment transport and morphodynamics (e.g. Dubarbier et al. [8] ).

\subsection{Bichromatic waves}

In this section, the ability of our shallow water formulas to reconstruct nonlinear waves is assessed with respect to a bichromatic wave field propagating over a gently sloping $(1 / 20)$ movable bed. The small-scale experimental set-up is described in Michallet et al. [23] and references therein. The two frequencies composing the wave-board motion were $f_{1}=0.5515 \mathrm{~Hz}$ and $f_{2}=0.6250 \mathrm{~Hz}$, and the amplitude of the two wave components were identical with a value of $0.03 \mathrm{~m}$. Water elevation and bottom pressure were synchronously measured (at 


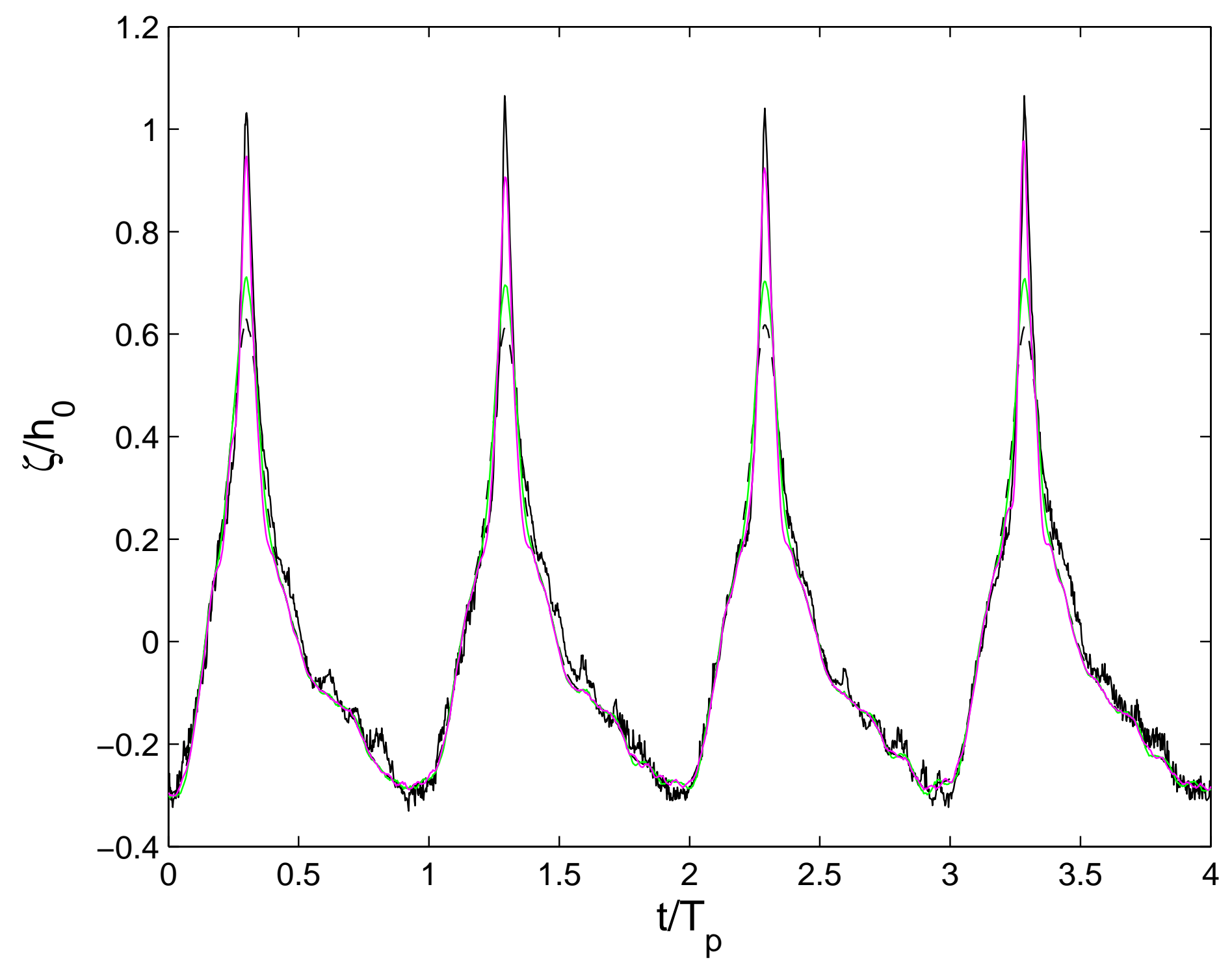

Figure 2: Surface elevation reconstruction of monochromatic waves. A7-mono test obtained during BARDEXII, $h_{0}=1.17 \mathrm{~m}, T_{p}=12.1 \mathrm{~s}$ and $\delta_{m}=0.33 \mathrm{~m}$. Dimensionless cut-off frequency $T_{p} f_{c}=20$. black line: direct LiDAR measurement of $\zeta$; dashed black line: hydrostatic reconstruction $\zeta_{\mathrm{H}}$, Eq. 11); green line: $\zeta_{\mathrm{SL}}$, Eq. 12); magenta line: $\zeta_{\mathrm{SNL}}$, Eq. 131). 


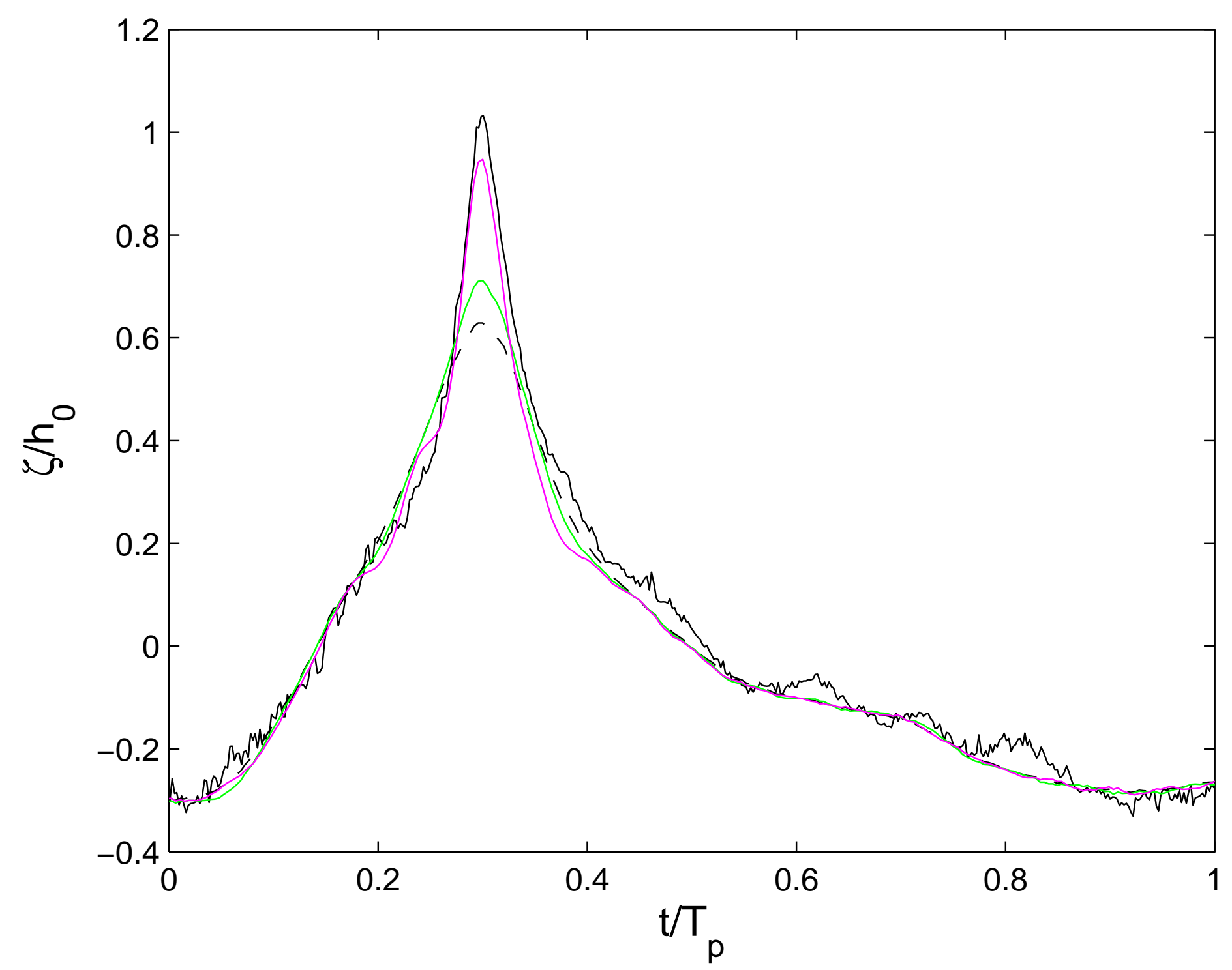

Figure 3: Surface elevation reconstruction of monochromatic waves. Zoom over one period of A7-mono test obtained during BARDEXII, $h_{0}=1.17 \mathrm{~m}, T_{p}=12.1 \mathrm{~s}$ and $\delta_{m}=0.33$ m. Dimensionless cut-off frequency $T_{p} f_{c}=20$. black line: direct LiDAR measurement of $\zeta$; dashed black line: hydrostatic reconstruction $\zeta_{\mathrm{H}}$, Eq. (11); green line: $\zeta_{\mathrm{SL}}$, Eq. (12); magenta line: $\zeta_{\mathrm{SNL}}, \mathrm{Eq}$. [13). 

tion was monitored with a capacitive wave gauge. Its accuracy was estimated to be of approximately $1 \mathrm{~mm}$ from comparison with high-speed video recordings. The pressure sensor was located at the bed. The still water depth at the measurement location was $h_{0}=0.185 \mathrm{~m}$, the characteristic bottom slope was for the highest wave of the group, $\varepsilon=0.37$.

Figure 4 presents a comparison between shallow water reconstructions and direct elevation measurements. The nonlinear formula (13) significantly improves the elevation reconstruction compared with the linear formula (12), escrests in the second half of the wave packet. It was observed that the erosion depth and the mobile sediment layer thickness were enhanced after the highest wave. Further investigations would be needed to confirm how the sediment dynamics might contribute to damp high frequencies at the sensor location. density spectrum and the spectra obtained from reconstruction formulas. We can see that the linear (12) and nonlinear (13) reconstructions properly describe the elevation energy around the first (i.e. fundamental) harmonic. For higher harmonics, the agreement between the linear reconstruction (12) and di-

\subsection{Field data}

The data was collected during a field campaign performed on April 13-14 2017, at La Salie beach, situated on the southern part of the French Atlantic coast. Several instruments were deployed at low tide to characterize the shallow water wave field: a stereophotogrammetry system, a Nortek Signature 1000 


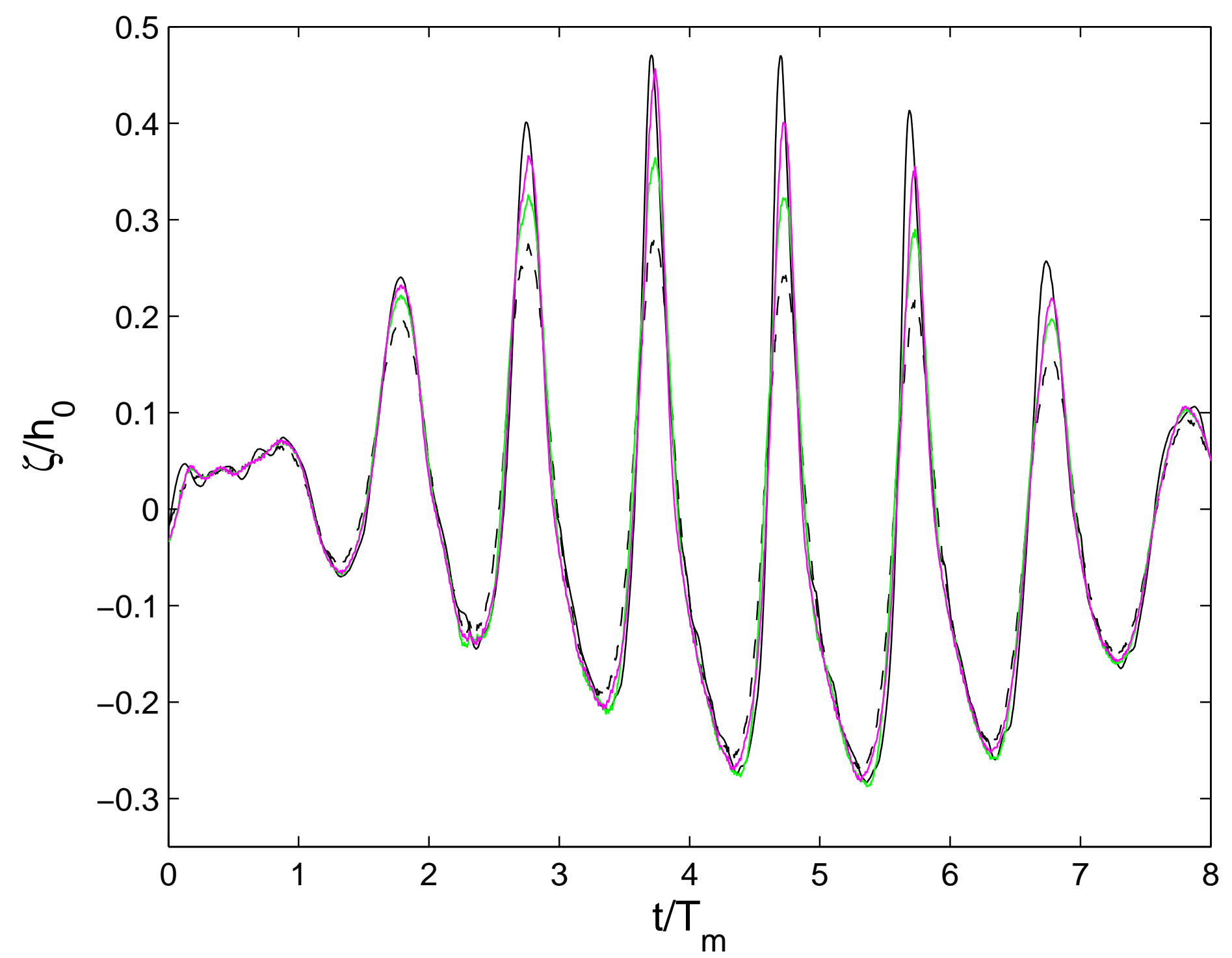

Figure 4: Surface elevation reconstruction of bichromatic waves, $f_{1}=0.5515 \mathrm{~Hz}, f_{2}=0.6250$ $\mathrm{Hz}\left(T_{m}=\left(\frac{f_{1}+f_{2}}{2}\right)^{-1}\right), h_{0}=0.185 \mathrm{~m}$ and $\delta_{m}=0$. Dimensionless cut-off frequency $T_{m} f_{c}=$ 4.5. black line: direct measurement of $\zeta$; dashed black line: hydrostatic reconstruction $\zeta_{\mathrm{H}}$, Eq. 111; green line: $\zeta_{\mathrm{SL}}$, Eq. 12); magenta line: $\zeta_{\mathrm{SNL}}$, Eq. 13. 


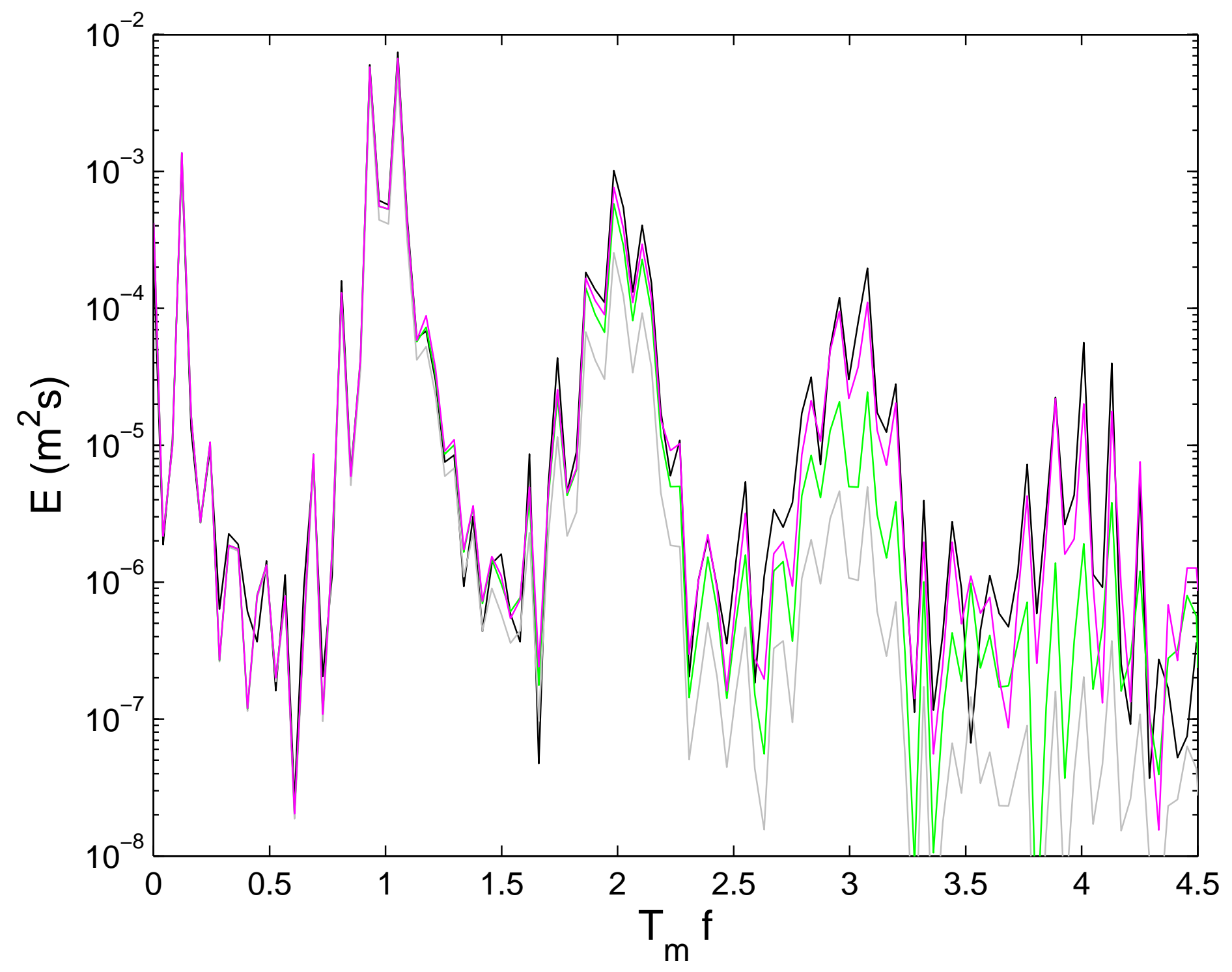

Figure 5: Surface elevation energy density spectra, $E(f)$, as a function of the dimensionless frequency $T_{m} f$, for bichromatic waves, $f_{1}=0.5515 \mathrm{~Hz}, f_{2}=0.6250 \mathrm{~Hz}\left(T_{m}=\left(\frac{f_{1}+f_{2}}{2}\right)^{-1}\right)$, $h_{0}=0.185 \mathrm{~m}$ and $\delta_{m}=0$. Dimensionless cut-off frequency $T_{m} f_{c}=4.5$. black line: direct measurement of $\zeta$; grey line: hydrostatic reconstruction $\zeta_{\mathrm{H}}$, Eq. 111; green line: $\zeta_{\mathrm{SL}}$, Eq. 12); magenta line: $\zeta_{\mathrm{SNL}}$, Eq. 13). 
(Ocean Sensor Systems). At the deployment location the characteristic bottom slope was $\sigma_{b}=0.015$. In the present paper we focus only on one 10-min data set (see Figure 6) corresponding to nonlinear shallow water waves. Together with bottom pressure measurements (10 Hz sampling rate), a direct measurement of $\mathrm{kHz}$ ( $8 \mathrm{~Hz}$ sampling rate). The video recording shows that the highest waves were breaking just shoreward of the sensors. Martins et al. 20] recently compared surface elevation measurements from LiDAR and a Signature $1000 \mathrm{kHz}$ in the Garonne River during a non-breaking undular tidal bore event. Despite in tracking the free surface and estimating individual wave heights ( $R M S$ error of $0.05 \mathrm{~m})$.

The wave conditions at the water depth $\bar{h}=2.25 \mathrm{~m}$ were characterized by a significant wave height of $0.70 \mathrm{~m}$, with a peak period of $11.1 \mathrm{~s}(\mu=0.075)$;

arge-amplitude wave groups were also observed (see Figure 6). The maximum wave height in these wave groups is $1.4 \mathrm{~m}$, which corresponds to a nonlinearity parameter $\varepsilon$ of 0.31 . Figure 7 shows that the linear reconstruction (12) gives good results for the lowest waves of the wave group but strongly underestimates the elevation at the crest of the highest waves. On the other hand, our nonlinear formula (13) gives excellent results even for the highest waves. A zoom around the highest wave of the wave group observed in Figure 7 is presented in Figure 8. We can see in this figure that the linear reconstruction fails to predict the maximum elevation and above all the skewed shape of this nonlinear wave. By contrast, these wave properties are very well reproduced by the nonlinear 225 and the spectra obtained from reconstruction formulas are compared in Figure 9. In agreement with observations for laboratory bichromatic waves (see Figure 5) we can see that a nonlinear method is required to properly reconstruct the surface elevation spectrum, especially for the highest harmonics. 


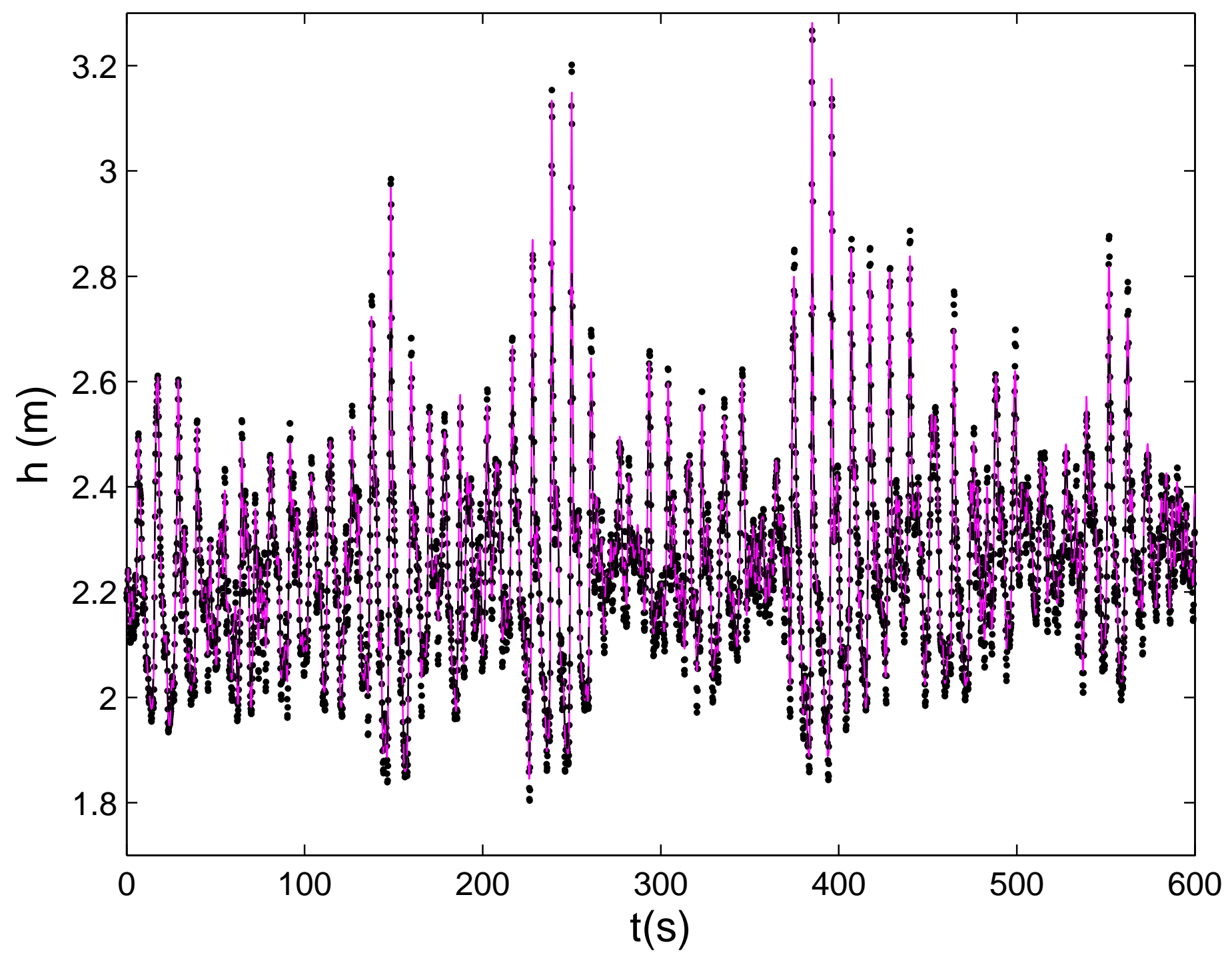

Figure 6: Reconstruction of water depth time series of waves observed in the field. Cut-off frequency $f_{c}=1 \mathrm{~Hz}, \bar{h}=2.25 \mathrm{~m}, \delta_{m}=0.69 \mathrm{~m}$. dot: direct acoustic measurement of $h$; magenta line: $h_{\mathrm{SNL}}=\bar{h}+\zeta_{\mathrm{SNL}}$, Eq. (13). 


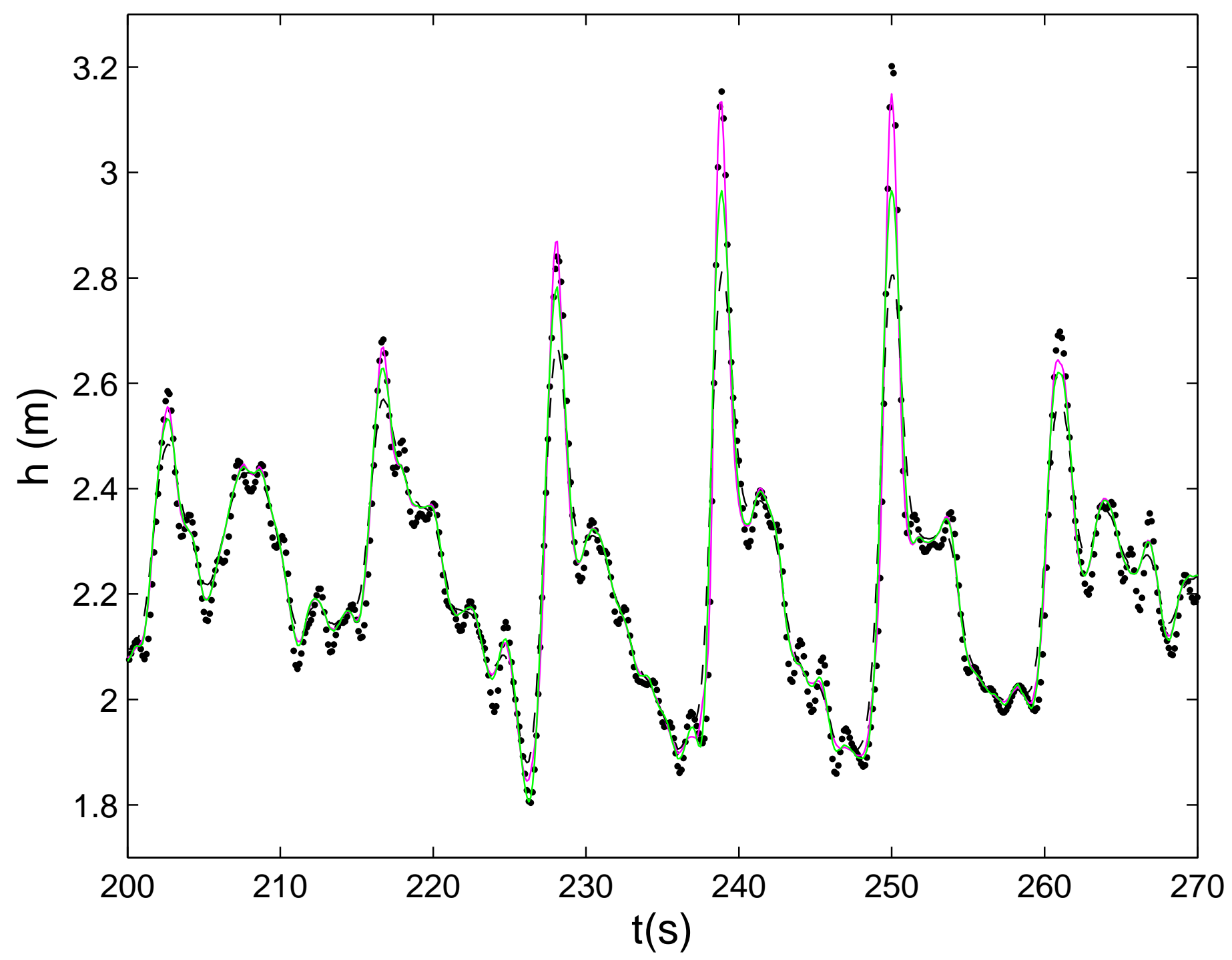

Figure 7: Reconstruction of water depth time series of a group of waves observed in the field. Cut-off frequency $f_{c}=1 \mathrm{~Hz}, \bar{h}=2.25 \mathrm{~m}, \delta_{m}=0.69 \mathrm{~m}$. dot: direct acoustic measurement of $h$; green line: $h_{\mathrm{SL}}=\bar{h}+\zeta_{\mathrm{SL}}$, Eq. 12); magenta line: $h_{\mathrm{SNL}}=\bar{h}+\zeta_{\mathrm{SNL}}$, Eq. 13). 


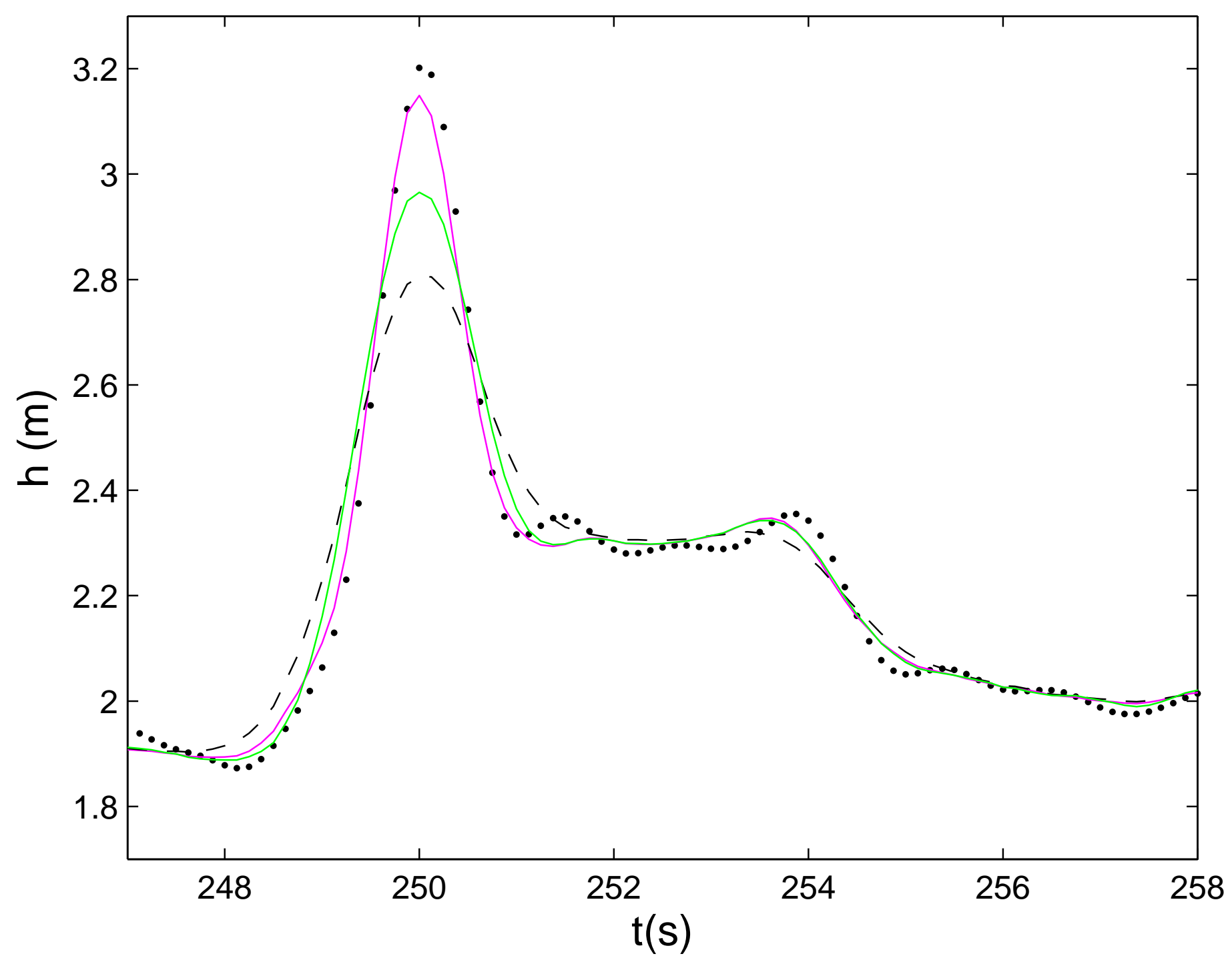

Figure 8: Reconstruction of the highest wave observed in a wave group. Cut-off frequency $f_{c}=1 \mathrm{~Hz}, \bar{h}=2.25 \mathrm{~m}, \delta_{m}=0.69 \mathrm{~m}$. dot: direct acoustic measurement of $h$; dashed black line: hydrostatic reconstruction $\zeta_{\mathrm{H}}$, Eq. (11); green line: $h_{\mathrm{SL}}=\bar{h}+\zeta_{\mathrm{SL}}$, Eq. (12); magenta line: $h_{\mathrm{SNL}}=\bar{h}+\zeta_{\mathrm{SNL}}$, Eq. 13). 


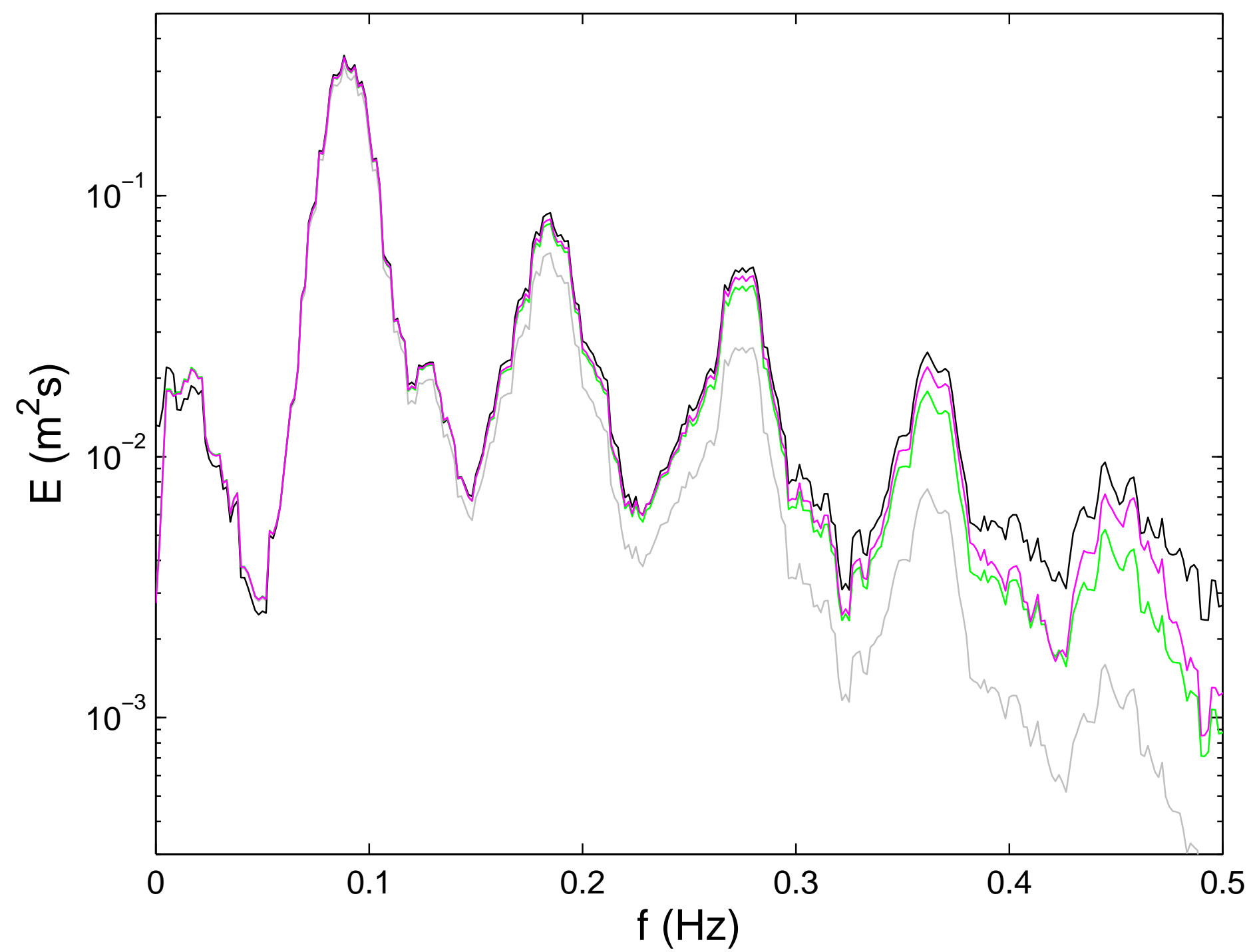

Figure 9: Surface elevation energy density spectra, $E(f)$, of waves observed in the field. The spectra have been smoothed with a moving average over a window of $1 / 60 \mathrm{~Hz}$. black line: direct measurement of $\zeta$; grey line: hydrostatic reconstruction $\zeta_{\mathrm{H}}$, Eq. (11); green line: $\zeta_{\mathrm{SL}}$, Eq. (12); magenta line: $\zeta_{\mathrm{SNL}}$, Eq. 13. 


\section{Conclusion}

We have derived a nonlinear weakly dispersive formula (Eq. (13)) to reconstruct, from pressure measurements, the surface elevation of nonlinear waves propagating in shallow water. This simple and easy to use formula is local in time, only involving first and second order time derivatives of the measured pressure $P_{\mathrm{m}}$, or equivalently $\zeta_{\mathrm{H}}$. Contrary to fully dispersive reconstruction formulas it does not necessarily require Fourier transform. The ability of this formula to reconstruct surface elevation has been assessed from laboratory and field experiments of weakly dispersive waves $(\mu \leq 0.28)$ propagating in the shoaling zone just prior to breaking. Despite the strong nonlinearities naturally found close to the breaking point, and even in the presence of strong wave reflection (Section 3.1), the novel method was found to perform very well.

We have shown that the nonlinear formula (13) provides much better reconstructed surface elevation than linear methods. Our nonlinear reconstruction is able to accurately reproduce the peaked and skewed shape of nonlinear waves prior to breaking. Although the linear reconstruction method properly describes the surface elevation energy density around the fundamental harmonic, it strongly underestimates the energy density for the higher harmonics. In contrast, the nonlinear weakly dispersive reconstruction (13) accurately predicts the surface wave elevation spectrum over four harmonics. This demonstrates that a nonlinear approach is essential for nonlinear wave reconstruction in the shoaling zone and especially near the breaking point. However, the derivation of our nonlinear formula (Eq. (13) ) is based on a shallowness assumption. For nonlinear waves propagating in intermediate water depth a fully dispersive nonlinear reconstruction is required (see Bonneton and Lannes [6]).

55 An accurate description of surface wave elevation is crucial for many coastal applications, such as wave-induced sediment transport or overtopping and submersion associated with extreme waves. In this context, the application of nonlinear reconstruction methods on pressure data represent an economic and easy to use alternative to direct wave elevation measurement methods, such as 
circumstances.

\section{Acknowledgments}

Philippe Bonneton and David Lannes have been partially funded by the ANR-13-BS01-0009-01 BOND. David Lannes also acknowledges support from the ANR-13-BS01-0003-01 DYFICOLTI, Philippe Bonneton from the ANR-14ASTR-0019 COASTVAR and Kévin Martins from the University Research Services scholarship from University of Bath. The BARDEXII data set used in this study is available from http://www.hydralab.info, and it was obtained with the support of the European Community's 7th Framework Programme through the grant to the budget of the Integrating Activity HYDRALAB IV, contract no. 261520. The authors are grateful to all those who contributed to the field experiment at La Salie Beach: Bonneton N., Castelle B., Detandt G., Dubarbier B., Marieu V. and Poncet P.A..

\section{Appendix A. Local time discretization method for the surface recon- struction}

In contrast to fully dispersive reconstructions, the weakly dispersive methods derived in the present paper are local in time and thus do not necessarily require the use of Fourier transforms. However, to accurately compute time derivatives and to easily filter out the measurement noise we have used Fourier transforms in this paper (see section 3). When the measured pressure time series is too short for a Fourier analysis a local time discretization method is required. The time derivatives involved in equations (12) and (13) can be computed, at order $O\left(\Delta t^{2}\right)$, as follows

$$
\begin{aligned}
\left.\partial_{t} \alpha\right|^{n} & =\frac{\alpha^{n+1}-\alpha^{n-1}}{2 \Delta t} \\
\left.\partial_{t}^{2} \alpha\right|^{n} & =\frac{\alpha^{n+1}-2 \alpha^{n}+\alpha^{n-1}}{\Delta t^{2}} \\
\left.\partial_{t}\left(\alpha \partial_{t} \alpha\right)\right|^{n} & =\frac{\alpha^{n+1}\left(\alpha^{n+2}-\alpha^{n}\right)-\alpha^{n-1}\left(\alpha^{n}-\alpha^{n-2}\right)}{4 \Delta t^{2}}
\end{aligned}
$$


where $\alpha^{n}$ denotes the discrete value of a flow variable $\alpha(t)$ at $t=n \Delta t$ and

$\Delta t$ is the measurement time step. This method properly reconstructs the wave field (see figure A.10), except that the measurement noise induces some spurious oscillations (see green line). This drawback is easily overcome (see blue line in figure A.10) by applying a moving average filter to the pressure measurements. The local method gives similar results than the Fourier one, except at the wave 290 crest where the Fourier method is more accurate. 


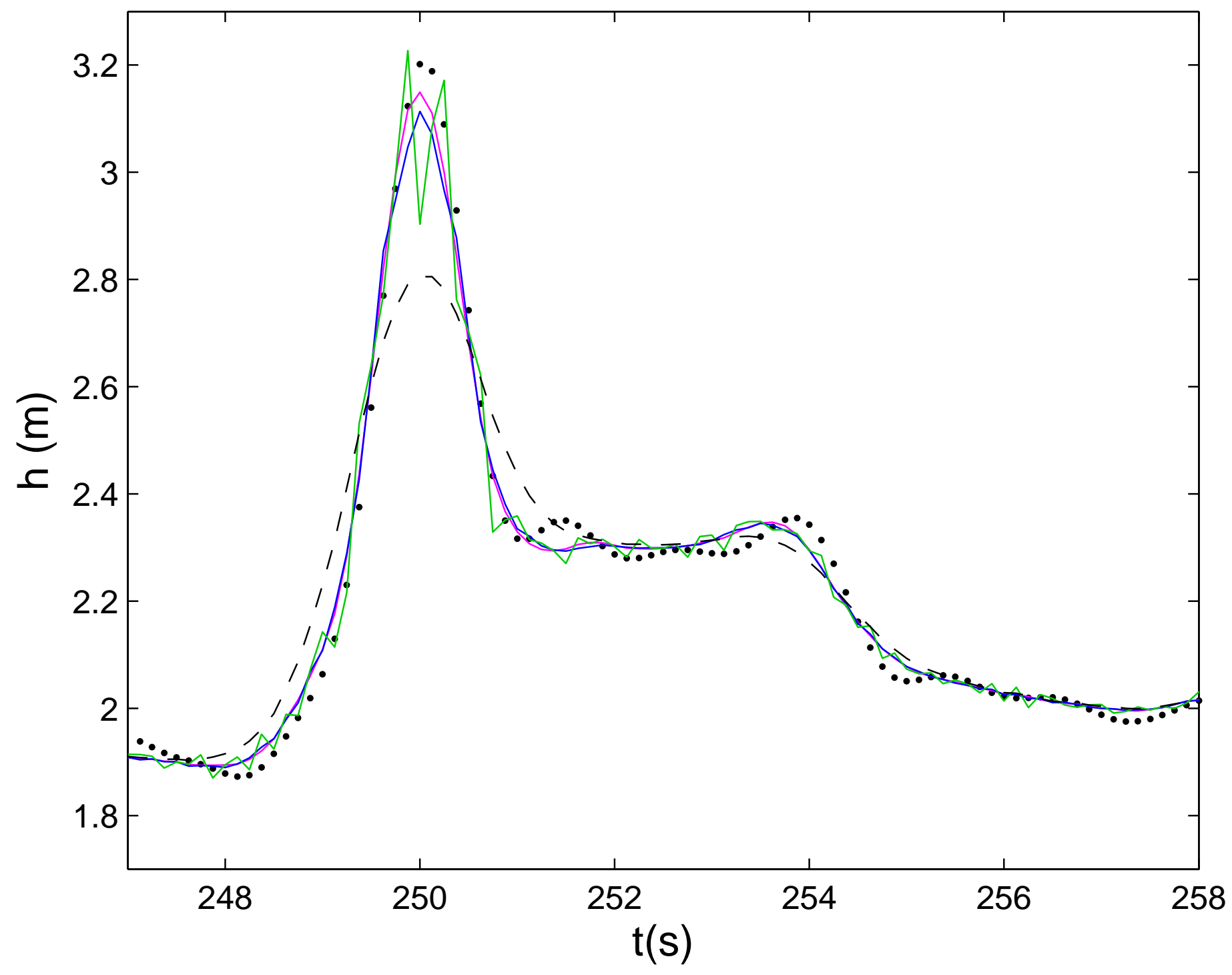

Figure A.10: Reconstruction of waves observed at La Salie beach (see section 3.3). $\bar{h}=$ $2.25 \mathrm{~m}, \delta_{m}=0.69 \mathrm{~m}$. dot: direct acoustic measurement of $h$; dashed black line: hydrostatic reconstruction $\zeta_{\mathrm{H}}$, Eq. (11); magenta line: $h_{\mathrm{SNL}}=\bar{h}+\zeta_{\mathrm{SNL}}$, Eq. (13) computed with a Fourier transform; green line: $h_{\mathrm{SNL}}=\bar{h}+\zeta_{\mathrm{SNL}}$, Eq. 13) computed with the local method A.1, without measurement noise filtering; blue line: $h_{\mathrm{SNL}}=\bar{h}+\zeta_{\mathrm{SNL}}$, Eq. 13) computed with the local method A.1 with a moving average filter of $\zeta_{\mathrm{H}}$ over $0.5 \mathrm{~s}$. 


\section{References}

[1] Abreu, T., Michallet, H., Silva, P.A., Sancho, F., van der A, D. and Ruessink, B.G. (2013). Bed shear stress under skewed and asymmetric oscillatory flows Coastal Engineering, 73, 1-10.

[2] Berni, C., Michallet, H. and Barthélemy, E. (2017). Effects of horizontal pressure gradients on bed destabilization under waves J. Fluid Mech., $\mathbf{8 1 2}$, 721-751.

[3] Birch, R., Fissel, D. B., Borg, K., Lee, V., and English, D. (2004). The capabilities of Doppler current profilers for directional wave measurements in coastal and nearshore waters. In OCEANS'O4. MTTS/IEEE TECHNOOCEAN'04, 3, 1418-1427.

[4] Bishop, C. T., and Donelan, M. A. (1987). Measuring waves with pressure transducers. Coastal Engineering, 11(4), 309-328.

[5] Bonneton, P., Barthelemy, E., Chazel, F., Cienfuegos, R., Lannes, D., Marche, F., and Tissier, M. (2011). Recent advances in Serre-Green Naghdi modelling for wave transformation, breaking and runup processes. European Journal of Mechanics-B/Fluids, 30(6), 589-597.

[6] Bonneton, P. and Lannes, D. (2017). Recovering water wave elevation from pressure measurements. Journal of Fluid Mechanics, 833, 399-429.

[7] Cavaleri, L. and Ewing, J. A. and Smith, N. D. (1978), Measurement of the Pressure and Velocity Field Below Surface Waves, In: Turbulent Fluxes Through the Sea Surface, Wave Dynamics, and Prediction; Eds. Favre, A. and Hasselmann, Klaus; Springer US, Boston, MA; pp. 257-272, 10.1007/978-1-4612-9806-9_17.

315 [8] Dubarbier, B., Castelle, B., Marieu, V. and Ruessink G. (2015). Processbased modeling of cross-shore sandbar behavior Coastal Engineering, 95, $35-50$. 
[9] Elgar, S., and Guza, R. T. (1985). Observations of bispectra of shoaling surface gravity waves. Journal of Fluid Mechanics, 161, 425-448.

320 [10] Elgar, S., Guza, R.T., Raubenheimer, B., Herbers, T. H. C. and Gallagher, E. L. (1997), Spectral evolution of shoaling and breaking waves on a barred beach, J. Geophys. Res., 102(C7), 15797-15805, doi:10.1029/97JC01010.

[11] Esteva, D., and Harris, D. (1970), Comparison of pressure and staff wave gage records, Proceedings of the 12th Coastal Engineering Conference, Washington, D.C., pp. 101-116.

[12] Guza, R. T., and Thornton, E.B. (1980), Local and shoaled comparisons of sea surface elevations, pressures, and velocities, J. Geophys. Res., 85(C3), 1524-1530, doi:10.1029/JC085iC03p01524.

[13] Henderson, S. M., Guza, R. T., Elgar, S., Herbers, T. H. C., and Bowen, A. J. (2006). Nonlinear generation and loss of infragravity wave energy. Journal of Geophysical Research: Oceans, 111(C12).

[14] Hom-ma, M., Horikawa, K., and Komori, S. (1966), Response characteristics of underwater wave gauge, Proceedings of the 10th Coastal Engineering Conference, Tokyo, Japan, pp. 99-114.

335 [15] Lannes, D. (2003), Secular growth estimates for hyperbolic systems. Journal of Differential Equations, 190, 466-503.

[16] Lannes D., Bonneton P. (2009) Derivation of asymptotic two-dimensional time-dependent equations for surface water wave propagation, Physics of Fluids, 21 (1), 016601 (9 pages), DOI: 10.1063/1.3053183.

340 [17] Lannes, D. The Water Waves Problem: Mathematical Analysis and Asymptotics, volume 188 of Mathematical Surveys and Monographs. AMS, 2013.

[18] Martins, K., Blenkinsopp, C.E, Zang, Z., (2016). Monitoring Individual Wave Characteristics in the Inner Surf with a 2-Dimensional 
Laser Scanner (LiDAR). Journal of Sensors, Article ID 7965431. DOI: $10.1155 / 2016 / 7965431$.

[19] Martins, K., Blenkinsopp, C.E, Almar, R., Zang, Z., (2017). On the influence of swash-based reflection on surf zone hydrodynamics: a wave-by-wave approach. Coastal Engineering, 122, 27-43.

[20] Martins, K., Bonneton, P., Frappart, F., Detandt, G., Bonneton, N. and Blenkinsopp, C.E (2017). High frequency field measurements of an undular bore using a 2D LiDAR scanner. Remote Sensing, 9(5), 462; doi:10.3390/rs9050462 .

[21] Martins, K., Blenkinsopp, C.E, Power, H.E. Bruder, B., Puleo, J.A., Bergsma, E.W.J., (2017). High-resolution monitoring of wave transformation in the surf zone using a LiDAR scanner array. Coastal Engineering, 128, 37-43.

[22] Masselink, G., Ruju, A., Conley, D., Turner, I., Ruessink, G., Matias, A.,Thompson C., Castelle B., Puelo J., Citerone V. and Wolters, G. (2016). Large-scale Barrier Dynamics Experiment II (BARDEX II): Experimental design, instrumentation, test program, and data set. Coastal Engineering, 113, 3-18.

[23] Michallet, H., Barthélemy, E., Lammens, A., Marin, G. and Vaudelin, G. (2017). Bed motion under waves: plug and sheet flow observations. Coastal Dynamics 2017, Helsingør, Denmark, paper No. 255.

[24] Oliveras, K. L., Vasan, V., Deconinck, B., and Henderson, D. (2012). Recovering the water-wave profile from pressure measurements. SIAM Journal on Applied Mathematics, 72(3), 897-918.

[25] Rocha, M. V. L., Michallet, H., and Silva, P. A. (2017). Improving the parameterization of wave nonlinearities - The importance of wave steepness, spectral bandwidth and beach slope. Coastal Engineering, 121, 77-89. 
[26] Senechal, N., Bonneton, P., and Dupuis, H. (2002). Field experiment on secondary wave generation on a barred beach and the consequent evolution of energy dissipation on the beach face. Coastal Engineering, 46(3), 233-247.

[27] Shi, F., Kirby, J. T., Harris, J. C., Geiman, J. D., and Grilli, S. T. (2012). A high-order adaptive time-stepping TVD solver for Boussinesq modeling of breaking waves and coastal inundation. Ocean Modelling, 43, 36-51.

[28] Tsai, C. H., Huang, M. C., Young, F. J., Lin, Y. C., and Li, H. W. (2005). On the recovery of surface wave by pressure transfer function. Ocean Engineering, 32(10), 1247-1259.

[29] Vasan, V., Oliveras, K., Henderson, D., and Deconinck, B. (2017). A method to recover water-wave profiles from pressure measurements. Wave Motion, 75, 25-35.

[30] Zijlema, M., Stelling, G., and Smit, P. (2011). SWASH: An operational public domain code for simulating wave fields and rapidly varied flows in coastal waters. Coastal Engineering, 58(10), 992-1012. 